\title{
Certified Pediatric Oncology Nurse
}

National Cancer Institute

\section{Source}

National Cancer Institute. Certified Pediatric Oncology Nurse. NCI Thesaurus. Code C71331.

A licensed Registered Nurse who has achieved the recognition of meeting criteria necessary to be specialized in the field of Pediatric Oncology, including working at least 1 year within the past 3 years with at least 1,000 hours spent in Pediatric Oncology Nursing within the past $21 / 2$ years. In addition, at least 10 continuing education hours in the field of Pediatric Oncology Nursing should have been completed over the past 3 years. 